Pensar en Movimiento: Revista de Ciencias del Ejercicio y la Salud. Vol. 6, Nº1, 2008, ISSN 1659-4436

Adulto Mayor: Ejercicio y Hemodinámica

\title{
Un programa de ejercicio contra resistencia estimula cambios positivos en la respuesta hemodinámica en adultos mayores
}

\author{
Cinthya Campos Salazar, M.Sc. ${ }^{1}$ y Norbel Román Garita, $M D^{2}$ \\ ${ }^{1}$ Escuela de Educación Física y Deportes, Universidad de Costa Rica \\ ${ }^{2}$ Hospital Gerontológico Dr. Raúl Blanco Cervantes, San José, Costa Rica \\ E-mail: cinthia.campos@ucr.ac.cr \\ Manuscrito recibido: 02/10/08. Aceptado: 25/02/09
}

\begin{abstract}
RESUMEN
Campos-Salazar, C., y Román-Garita, N. (2008). Un programa de ejercicio contra resistencia estimula cambios positivos en la respuesta hemodinámica en adultos mayores. PENSAR EN MoviMIENTO: Revista de Ciencias del Ejercicio y la Salud, 6(1), 13-23. El propósito del estudio fue determinar el efecto de un programa de ejercicios contra resistencia en las respuestas y adaptaciones hemodinámicas de adultos mayores de 60 años. Se reclutaron 60 adultos mayores sanos, quienes ejecutaron un programa de entrenamiento de 3 sesiones semanales durante 12 semanas. Los participantes fueron asignados aleatoriamente a un grupo control, a un grupo que se ejercitó al 30\% de 5 repeticiones máximas (5RM) o a un grupo que se ejercitó al $70 \%$ de 5RM. Las variables hemodinámicas fueron la presión arterial media (PAM), la cual fue calculada antes e inmediatamente después de realizar el ejercicio, y el doble producto (DP), que se determinó una vez cada 4 semanas durante el programa y también antes y después de finalizado el mismo. Los resultados mostraron que el ejercicio contra resistencia al 30\% y 70\% de 5RM, con una carga total de ejercicio entre 872.7 y 890.9 kg, respectivamente, no presenta niveles de riesgo cardiovascular. Se observó que el programa de 12 semanas de entrenamiento contra resistencia reduce el grado de estrés cardiovascular medido a través del DP ( 16\%) y la PAM ( 9\%), sin presencia de eventos adversos durante todo el programa. Lamentablemente, los beneficios hemodinámicos se revirtieron a los 6 días de finalizado el programa de ejercicios. En conclusión, la población adulta mayor sana debe realizar un programa de actividad física constante para reducir significativamente el grado de estrés cardiovascular. Se recomienda realizar investigaciones con las mismas intensidades de ejercicio en programas de mayor duración y determinar el proceso de pérdida de la adaptación en otras variables fisiológicas, bioquímicas y biomecánicas.
\end{abstract}

PALABRAS CLAVES: entrenamiento contra resistencia, adulto mayor, presión arterial, frecuencia cardíaca.

\begin{abstract}
Campos-Salazar, C., y Román-Garita, N. (2008). A 12-week resistance training program elicits positive changes in hemodynamic responses in the elderly. Pensar en MovimiEnto: Revista de Ciencias del Ejercicio y la Salud, 6(1), 13-23. The aim of the study was to determine the effect of a resistance training program in hemodynamic responses and adaptations in 60 yr. old elderly. Volunteers were 60 healthy-elderly who underwent a training program 3 times/wk. for $12 \mathrm{wk}$. Participants were randomly assigned to either a control group, an exercise group who trained at 30\% intensity of 5 maximal repetitions (5RM) (30\% of 5RM) or an exercise group at an intensity of $70 \%$ (70\% of 5RM). Hemodynamic variables measured were mean arterial pressure (MAP), calculated before and immediately after the training session, and rate pressure product (RPP), estimated once a month and before and after finishing the program. Results indicated that resistance exercise training at 30\% and 70\% of 5RM, with a total exercise work of 872.7 and $890.9 \mathrm{~kg}$ did not elicited cardiovascular risks for the elderly. A 12-wk resistance exercise training reduced the cardiovascular strain as shown by the RPP ( 16\%) and the MAP ( 9\%), with no adverse effects throughout the program. Unfortunately, all the hemodynamic benefits were reverted 6 days following completion of the program. In conclusion, a healthy elderly population must perform resistance training exercises to significantly reduce the cardiovascular stress. We suggest to conduct further research that looks into different exercise intensities in
\end{abstract}


Pensar en Movimiento: Revista de Ciencias del Ejercicio y la Salud. Vol. 6, No1, 2008, ISSN 1659-4436

Adulto Mayor: Ejercicio y Hemodinámica

longer program duration and to determine the mechanisms responsible for the deleterious effects of the detraining by using physiological, biochemical and biomechanical variables.

KEY WORDS: resistance training, elderly, blood pressure, heart rate.

El proceso de envejecimiento de los seres vivos es en general, universal, constante e irreversible, pero varía de acuerdo a la especie, o bien, a su propio ritmo de envejecimiento interno. El ser humano no escapa a esta realidad absoluta $\mathrm{y}$ enfrenta un proceso de envejecimiento tanto en sus órganos, como en su composición química, física y por consiguiente en su funcionamiento (Miller, 1994). Lo que sí es claro en todas las especies, es que el envejecimiento es el proceso que lleva a la falla de la adaptación al medio ambiente y que finalmente, resulta en la muerte.

Existe evidencia sistemática que indica el beneficio de ejercicios de diversas modalidades en el adulto mayor (Green \& Crouse, 1995; Braith \& Stewart, 2006). Los beneficios incluyen cambios anatómicos y funcionales, así como mejores perfiles fisiológicos y cognitivos (Heyn, Abreu \& Ottenbacher, 2004; Teunissen, de Jonge, van Weel \& Lagro-Janssen, 2004; Lopopolo, Greco, Sullivan, Craik \& Mangione, 2006;). Estos beneficios le permiten a la persona adulta mayor obtener una mayor independencia y reducir el riesgo de lesiones (Province, Hadley, Hornbrook, Lipsitz, Philip, Mulrow, Ory, Sattin, Tinetti \& Wolf, 1995; HillWestmoreland, Soeken \& Spellbring, 2002). Actualmente, existe clara evidencia de que el ejercicio contra resistencia (pesas) afecta positivamente el sistema óseo y muscular durante el proceso de envejecimiento. Se han descrito aumentos significativos en la fuerza de diferentes grupos musculares en personas mayores luego de realizar ejercicio contra resistencia (Brown, Cartney, \& Sale, 1981; Mac Dougall, 1985; Spiliä \& Suominen, 1995; Kaver, Fonseca y Salazar, 1999; Madden, Levy \& Stratton, 2006).

Dos de los reportes más impresionantes que describen el efecto del entrenamiento en el adulto mayor son el de Frontera et al. (1988) y Fiatarone et al. (1990), quienes encontraron un aumento en la fuerza muscular de los músculos flexores y extensores de la pierna de entre $107 \%$ y $227 \%$ en hombres entre los 60 y 90 años de edad. Con resultados como éstos se ha llegado al consenso de que la disminución de la fuerza muscular asociada con el envejecimiento no es debida únicamente a este proceso, sino también a la falta de estímulo externo no asociada a la reducción de la fuerza muscular que se presenta con el paso de los años.

Kaver y col. (1999), realizaron un estudio en el cual compararon el efecto de dos intensidades distintas, 30\% de 5 RM y 70\% de 5 RM en la fuerza muscular de personas mayores de 60 años; la diferencia con los estudios previamente citados es que compara el efecto de intensidades distintas, y controla el volumen total de ejercicio por sesión. Los resultados mostraron un aumento significativo en la fuerza muscular a ambas intensidades y no hubo diferencia entre grupos de ejercicio.

$\mathrm{Al}$ igual que a nivel muscular, existen cambios a nivel cardiovascular asociados al envejecimiento. Particularmente, los niveles de presión arterial y la frecuencia cardiaca no se justifican en su totalidad como producto de la vejez únicamente. Por ejemplo, en la literatura se reporta que la presión arterial en reposo aumenta con la edad y una presión arterial elevada en reposo es un factor de riesgo de enfermedad principal en el adulto mayor (Joint National Committee on Prevention, Detection, Evaluation, and Treatment of High Blood Pressure, 2003).

Grupos de investigadores han intentado comprobar si el ejercicio contra resistencia tiene algún efecto sobre variables cardiovasculares y hemodinámicas. Como respuesta aguda al ejercicio diversos investigadores (MacDougall, Tuxen, Sale, Moroz, \& Sutton, 1985; Fleck, 1988; Stone, Fleck, Triplett \& Kraemer, 1991), han indicado que la frecuencia cardiaca aumenta sustancialmente como respuesta al ejercicio contra resistencia con cargas altas. Se considera una carga alta, un ejercicio en el que se sobrepase el $85 \%$ de una repetición máxima (RM). Un RM se define como aquella resistencia que se pueda vencer una vez. MacDougal et al. (1985), encontraron que los participantes que ejecutaron el "press" o prensa de piernas hasta el fallo positivo o agotamiento muscular de acciones concéntricas al 95\% de 1RM o fuerza concéntrica máxima, alcanzaron pulsaciones de 170 latidos por minuto $\left(\right.$ lat $\bullet \mathrm{min}^{-1}$ ) y reportaron, además, que los 
Pensar en Movimiento: Revista de Ciencias del Ejercicio y la Salud. Vol. 6, No1, 2008, ISSN 1659-4436

Adulto Mayor: Ejercicio y Hemodinámica

valores más altos de la frecuencia cardiaca se alcanzan en las últimas repeticiones, debido al aumento en la activación de la masa muscular.

Sin embargo, Hagerman et al. (2000), diseñaron un estudio en que participaron adultos mayores no entrenados con edades entre 60 y 75 años para determinar el efecto de un programa de entrenamiento contra resistencia de alta intensidad. El programa tuvo una duración de 16 semanas y cada sesión estuvo separada por $48 \mathrm{~h}$. El programa consistió de tres series hasta el fallo con una carga de 85 a $90 \%$ de 1RM. Los ejercicios realizados fueron el press de piernas, media sentadilla y extensión rodilla con uno a dos minutos de descanso entre series. Los resultados del estudio indicaron que no se encontraron cambios en la frecuencia cardiaca pico ni en la presión arterial, por lo que se concluye que los adultos mayores, varones, toleran cargas de intensidad altas al realizar ejercicio contra resistencia.

Así, se espera que la persona adulta mayor también muestre adaptaciones al ejercicio. Stone et al. (1991) indican que los atletas que entrenan con cargas altas tienen menor $\mathrm{o}$ igual frecuencia cardiaca en reposo que el promedio normal. En adultos mayores, Madden et al. (2006) indican que el ejercicio contra resistencia no tiene efecto sobre la variabilidad de la frecuencia cardiaca, un indicador del grado de activación del sistema electroquímico y nervioso del corazón. Son pocos los estudios que han determinado este efecto, por lo cual se requiere de más investigación para ratificar la adaptación del ejercicio contra resistencia sobre esta variable en la población mayor.

Actualmente se sabe que el ejercicio de tipo aeróbico reduce los niveles de presión arterial sistólica y diastólica en reposo, tanto en personas adultas mayores normotensas, como hipertensas (Duncan et al., 1985; Hagberg, Montain, Martin \& Ehsani, 1989; Pescatello, Fargo, Leach, \& Scherzer, 1990). Sin embargo, por mucho tiempo se creyó que el ejercicio contra resistencia provocaba como adaptación una hipertensión arterial crónica; no obstante varios investigadores (Fleck, 1988; Goldberg, 1989; Stone et al., 1991), señalan una posición contraria, pues sostienen que programas de ejercicio contra resistencia por un período de 6 a 20 semanas de duración, no cambian o disminuyen los niveles de presión arterial sistólica y diastólica en reposo. También indican que los atletas que entrenan con cargas altas tienen similar o menor nivel que el promedio normal de presión arterial (Fleck, 1988; Goldberg, 1989; Stone et al., 1991).

En su momento, lo que originó la idea de que el ejercicio con pesas o contra resistencia causa hipertensión arterial crónica fue la presencia de este padecimiento en atletas que entrenaban bajo esa modalidad; pero según Stone et al. (1991), la presencia de dicha patología no se debía únicamente al entrenamiento, sino que se asociaba a la presencia de otros factores, como el uso de andrógenos que provocaban excesivas ganancias musculares, o bien, por un sobreentrenamiento crónico o la presencia de hipertensión arterial esencial.

Con base en esta evidencia, es importante determinar la variación de la presión arterial sistólica y diastólica y la frecuencia cardiaca en personas mayores de 60 años al realizar ejercicio con pesas o contra resistencia. Este planteamiento es relevante ya que podría comprobarse que además del beneficio que genera a nivel muscular el realizar ejercicio con pesas, y por consiguiente en la capacidad funcional física de la persona mayor de 60 años, podría también beneficiar a nivel cardiovascular por la evidencia citada.

Sin embargo, por la disposición fisiológica de la persona mayor a padecer de hipertensión arterial, podría pensarse que esta población presente mayor riesgo que las personas de menor edad, al experimentar elevación de la frecuencia cardiaca y presión arterial en forma inmediata al realizar ejercicio con pesas, pues por sus cambios asociados con el envejecimiento en estas dos variables podrían aumentar más de lo esperado según la carga asignada. Si además se considera que en la persona mayor la recuperación a un esfuerzo físico se prolonga (Israel, 1992), a la vez se extiende el riesgo por elevación súbita de la frecuencia cardiaca y presión arterial al realizar ejercicio con pesas.

En este sentido se aplicará el protocolo utilizado por Kaver et al. (1999), quienes realizaron una prueba submáxima para determinar las intensidades de trabajo reduciendo el riesgo al realizar las pruebas. El cual además reporta ganancia de fuerza muscular en población mayor de 60 años.

Con respecto a la adaptación al entrenamiento contra resistencia de la frecuencia cardiaca y presión arterial sistólica y diastólica, el objetivo es comprobar si al igual que con personas menores de 60 años, se presenta una tendencia a mejorar el acondicionamiento cardiovascular. 
Por lo tanto el propósito del estudio fue determinar los cambios hemodinámicos agudos y crónicos debidos al entrenamiento contra resistencia a intensidades diferentes, controlando el volumen de ejercicio total por sesión. Específicamente, se estudian los cambios agudos y crónicos de la frecuencia cardiaca (FC), presión arterial sistólica (PAS) y diastólica (PAD), presión arterial media (PAM) y doble producto (DP).

\section{METODOLOGÍA}

Participantes. En el estudio participaron voluntariamente 60 hombres y mujeres mayores de 60 años y libres de riesgo coronario y problemas articulares severos. Los participantes además no habían realizado ejercicio con pesas anteriormente, $\mathrm{y}$ presentaron nivel funcional alto en cuanto a marcha y equilibrio. Los valores de FC, PAS y $\mathrm{PAD}$ iniciales se muestran en las Figuras 1, 2 y 3 respectivamente.

Instrumentos de medición. Los instrumentos que se utilizaron fueron un esfigmomanómetro tipo aneroide y un estetoscopio tradicional (Prestige Medical $^{\circledR}$ ) para la medición de PAS y PAD y cronómetros para la FC. El equipo biomecánico fue la máquina de fuerza en banco horizontal y la máquina de extensión de rodillas.

\section{Procedimientos}

Reclutamiento y mediciones iniciales. Con el propósito de dar a conocer el estudio a la mayor cantidad de personas mayores, la divulgación general del programa y la fecha y hora del primer contacto se realizó por medio de avisos en el periódico de circulación nacional La Nación y mediante la Oficina de Divulgación de la Vicerrectoría de Acción Social de la Universidad de Costa Rica, quienes tienen nexo cercano con esta población debido al Programa de Tercera Edad (adulto mayor) que ofrecen.

A las personas que respondieron a la convocatoria se les explicó el objetivo e importancia del estudio y personal médico especialista en geriatría les realizó una entrevista y un electrocardiograma para completar la selección de la muestra de personas libres de riesgo coronario. Una vez seleccionados los participantes, se les solicitó el consentimiento formal de su participación voluntaria para el estudio. Posteriormente, en otra cita, se aplicaron las pruebas de PAS, PAD (método indirecto), y FC por el método palpatorio luego de 10 min previos de descanso.
Una vez realizado el registro de las mediciones iniciales se realizaron las pruebas de fuerza para cada uno de los ejercicios que conformaron el programa: a) fuerza en banco horizontal $y$ b) extensión de rodilla en máquina biomecánica. La prueba de fuerza consistió en realizar cinco repeticiones en cada uno de los ejercicios con la mayor carga posible, luego de un calentamiento los participantes procedían a ejecutar las 5 repeticiones iniciando con un peso de $6.8 \mathrm{~kg}$ (15 lb) para las mujeres y $13.6 \mathrm{~kg}$ (30 lb) para los hombres en el ejercicio de fuerza en banco horizontal y de $9.1 \mathrm{~kg}$ (20 lb) para las mujeres y $18.2 \mathrm{~kg}$ (40 lb) para los hombres en el ejercicio de extensión de rodillas.

Paulatinamente, con un descanso de dos minutos entre cada serie de 5 repeticiones, se aumentaban progresivamente $2.3 \mathrm{~kg}$ (5 lb) o $4.5 \mathrm{~kg}$ (10 lb) hasta que el sujeto no las completara, y entonces se registraba la carga anterior. La carga obtenida es lo que se conoce como 5 repeticiones máximas o 5 RM. La prueba de 5RM es una prueba submáxima que representa el $90 \%$ de $1 \mathrm{RM}$ y ha sido recomendada recientemente por Williams et al. (2007). Para el presente estudio por razones de seguridad muscular, articular y ósea, se determinó que $90 \%$ de la fuerza máxima era una carga aceptable por los cambios que presentan dichos sistemas por envejecimiento. Debido a las rápidas adaptaciones nerviosas que ocurren en personas no entrenadas, se decidió aumentar las cargas de trabajo después de 45 días con el propósito de cumplir con las intensidades de ejercicio planeadas a priori en este protocolo de entrenamiento.

Protocolo experimental. Luego de realizar las 5 RM iniciales, los participantes fueron asignados aleatoriamente a los tratamientos; es decir, a las dos intensidades de ejercicio y al grupo control. De esta manera, un grupo se ejercitó al 30\% de 5 RM (G30\%), es decir, al $27 \%$ de $1 \mathrm{RM}$, otro grupo se ejercitó al 70\% de 5 RM (G70\%), es decir, al 63\% de $1 \mathrm{RM}$, y el tercer grupo formó el grupo control (GCON). A los participantes del GCON se les indicó que mantuvieran su rutina de actividades habituales, con el objetivo de controlar el efecto de variables extrañas que pudieran incidir en las variables dependientes medidas a lo largo del programa.

La duración del programa fue de 12 semanas y los participantes asistieron 3 veces por semana a realizar las sesiones respectivas de ejercicio. Como se indicó anteriormente, los ejercicios que 
conformaron el programa fueron fuerza en banca horizontal, que fortalece los músculos pectorales, deltoides anterior y medio, y el tríceps braquial; y el ejercicio de extensión de rodillas, que fortalece el cuádriceps femoral.

El G30\% realizó 4 series de 16 repeticiones para cada ejercicio realizando una carga total de $872.7 \mathrm{~kg}$ (1920 lb) en cada ejercicio por día. El G70\% realizó en cada ejercicio 4 series de 7 repeticiones realizando una carga total de $890.9 \mathrm{~kg}(1960 \mathrm{lb})$, con lo cual el volumen de trabajo fue prácticamente idéntico en los dos grupos siendo la única diferencia la intensidad del ejercicio.

Diseño de investigación. Para determinar el efecto agudo el protocolo utilizado fue medir la PAS, PAD y FC antes de una sesión de ejercicios, con 10 minutos previos de descanso, y se midieron de nuevo inmediatamente después de la misma sesión de ejercicios. El protocolo se realizó una vez al mes durante los tres meses de duración del programa.

Para determinar el efecto crónico se midieron la PAS, PAD y FC, con 10 min previos de descanso, antes de iniciar el programa de ejercicios, una vez al mes durante los tres meses del programa y a la semana siguiente de finalizado el programa. Al GCON se le midió igualmente la PAS, PAD y FC con 10 min previos de descanso y permaneció en reposo por un lapso de 30 min, tiempo de duración de la sesión de ejercicios. El grupo control fue medido antes y después de finalizado el programa de ejercicios al mismo tiempo que los grupos experimentales.

Análisis estadístico. Se aplicó la técnica de análisis de varianza (ANOVA) de 3 vías mixta para los cambios agudos y crónicos de los grupos durante el ejercicio contra resistencia y una vez finalizado el mismo. Conociendo los cambios, se determinó la PAM y el DP, variables compuestas que permiten determinar el estrés al sistema cardiovascular y el consumo de oxígeno del corazón. Los análisis se realizaron con el Statistical Package for the Social Sciences, versión 8.0.

\section{RESULTADOS}

Las tres variables FC, PAS y PAD fueron medidas antes e inmediatamente después de la última serie de ejercicios de la sesión el día 30, 60 y 90 del programa. Los resultados de efecto agudo mostraron interacción significativa para la FC $\left(F_{(2,36)}=46.50, p<0.01\right)$, lo cual indica un aumento significativo de la FC inmediatamente después de realizar ejercicio contra resistencia, a pesar de que no hubo diferencia entre grupos de ejercicio (Figura 1).

De acuerdo con el ANOVA para la PAS, se encontró una interacción significativa de grupos y mediciones $\left(F_{(2,36)}=5.13, p<0.05\right)$. El cambio inmediato de la PAS fue diferente entre los grupos. El GCON no presentó cambios significativos en comparación con los grupos experimentales que realizaron las sesiones de ejercicio contra resistencia, los cuales aumentaron significativamente el nivel de PAS $\left(F_{(1,27)}=33.35, p\right.$ $<0.05)$. En este caso el incremento observado en el G70\% fue mayor que el aumento experimentado por el G30\% de 5 RM (Figura 2).

Con respecto a la PAD, el GCON como los grupos experimentales no presentaron cambio significativo en los niveles medidos antes e inmediatamente después de las sesiones del ejercicio contra resistencia (Figura 3). Adicionalmente, no se reportaron eventos negativos durante todo el programa de entrenamiento.

Con respecto al cambio por adaptación de las tres variables cardiovasculares en reposo se incluyeron 5 mediciones, iniciando con una toma antes de comenzar el programa, luego se realizó una medición cada mes del programa de ejercicios, en los días 30, 60, 90 y una última medición se realizó 6 días después de finalizado el programa. El análisis estadístico indicó que hubo un cambio significativo de la FC entre las mediciones $\left(F_{(4,108)}=3.15, p<\right.$ 0.05), disminuyendo paulatinamente a los 30 y 90 días del programa de ejercicios con respecto a la medición realizada antes de iniciar el programa, mientras que la medición realizada una vez finalizado el programa no fue significativamente diferente de la medición inicial. Este cambio ocurrió tanto en el G30\% como en el G70\%, y el patrón de cambio de los dos grupos experimentales no mostró diferencia significativa como se muestra en la Figura 4. En cuanto al grupo control no hubo cambios en la frecuencia cardiaca en reposo con respecto a la medición inicial. 


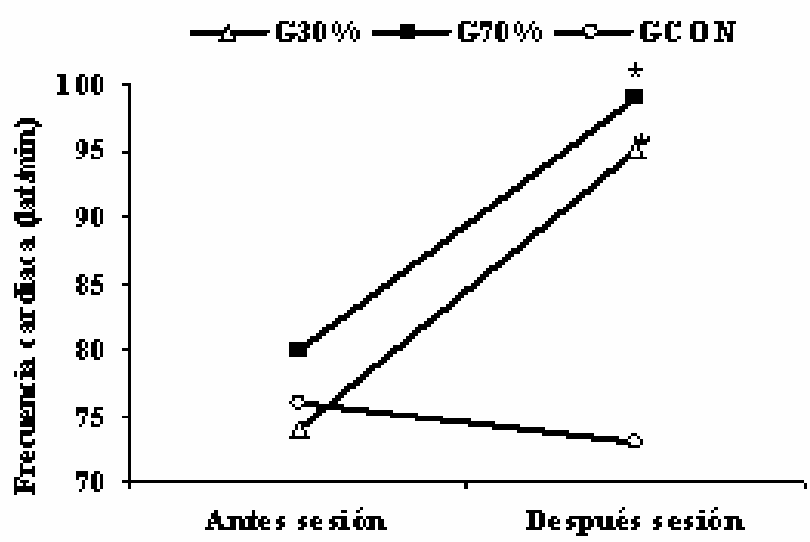

Figura 1. Respuesta aguda de la frecuencia cardiaca al realizar ejercicio contra resistencia.

${ }^{*} p<0.05$, con respecto a la medición antes del ejercicio

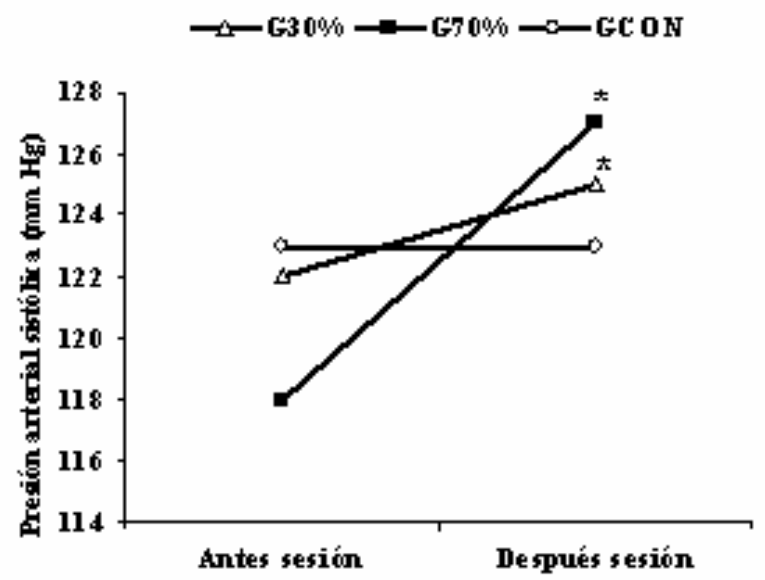

Figura 2. Respuesta de la presión arterial sistólica al realizar ejercicio contra resistencia.

La PAS en reposo también presentó una adaptación significativa $\left(F_{(4,108)}=12.04, p<0.01\right)$, disminuyendo a los 30, 60 y 90 días del programa de ejercicios con respecto a la medición realizada antes de iniciar el programa. Sin embargo, la medición realizada una vez finalizado el programa no fue significativamente diferente de la medición inicial. Al igual como ocurrió con la FC, no hubo diferencia significativa entre el patrón de cambio de los dos grupos que se ejercitaron. El GCON no presentó cambio significativo entre los niveles de presión arterial sistólica como se ilustra en la Figura 5.

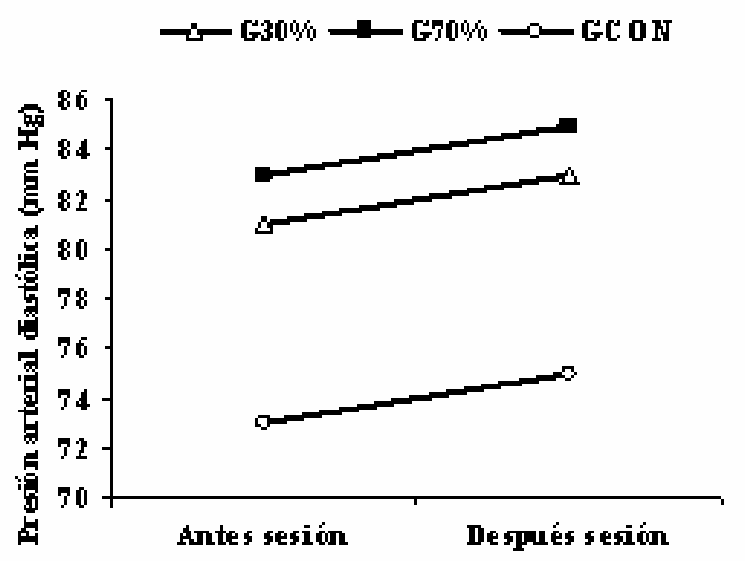

Figura 3. Respuesta de la presión arterial diastólica al realizar ejercicio contra resistencia.

La PAD cambió significativamente entre las mediciones a lo largo del programa $\left(F_{(4,108)}=4.43, p\right.$ $<0.01)$. Los niveles de PAD disminuyeron a los 30 , 60 y 90 días del programa de ejercicios con respecto a la medición realizada antes de iniciar el programa; y la medición realizada una vez finalizado el programa no fue significativamente diferente de la medición inicial. Igualmente que en las otras dos variables, no hubo diferencia significativa entre el patrón de cambio de los grupos. El GCON no presentó cambio significativo entre los niveles de presión arterial sistólica como se ilustra en la Figura 6.

\section{DISCUSIÓN}

El principal resultado de este estudio fue que el programa de ejercicio contra resistencia a dos intensidades distintas, con una duración de 12 semanas, no provocó valores pico en la PAM durante el ciclo cardiaco considerados de riesgo mientras se realizaba el ejercicio físico, estas mediciones se realizaron inmediatamente después de la última serie de ejercicios de tren superior.

Los valores de la PAM para los participantes del estudio oscilaron entre 91 y $99 \mathrm{~mm} \mathrm{Hg}$, tanto para los que se ejercitaron al 30\% como al $70 \%$ de intensidad (Figura 7); es decir, no sobrepasaron el rango normal de presión arterial media en las arterias, que oscila entre 93 y 107 mm Hg (Joint National Committee on Prevention, Detection, Evaluation, and Treatment of High Blood Pressure, 2003). Además un factor que pudo influir en estos resultados favorables, fue que se instruyó al 
participante acerca de la forma adecuada de la respiración con el fin de evitar la maniobra de Valsalva y durante la ejecución a cada participante se le reforzaba la técnica de respiración apropiada.

Este resultado puede explicarse debido a que las cargas de $30 \%$ y $70 \%$ se obtuvieron de la prueba de $5 R M$, que representan el $90 \%$ de la fuerza concéntrica máxima (1RM), por lo que las intensidades del estudio son menores que se si hubiesen obtenido de 1RM. Además, estos resultados coinciden con el estudio de Hagerman et al. (2000), quienes indican que hombres adultos mayores al realizar ejercicio contra resistencia a altas intensidades no presentaron cambios significativos en la frecuencia cardiaca pico ni en la presión arterial, mostrando tolerancia a nivel cardiovascular al realizar este tipo de ejercicio. De manera similar, el estudio de Bermon, Rama y Dolisi (2000) confirma también estos resultados con personas libres de cardiopatías.

- Control $\square \mathrm{G30} \% \square \mathrm{G} 70 \%$

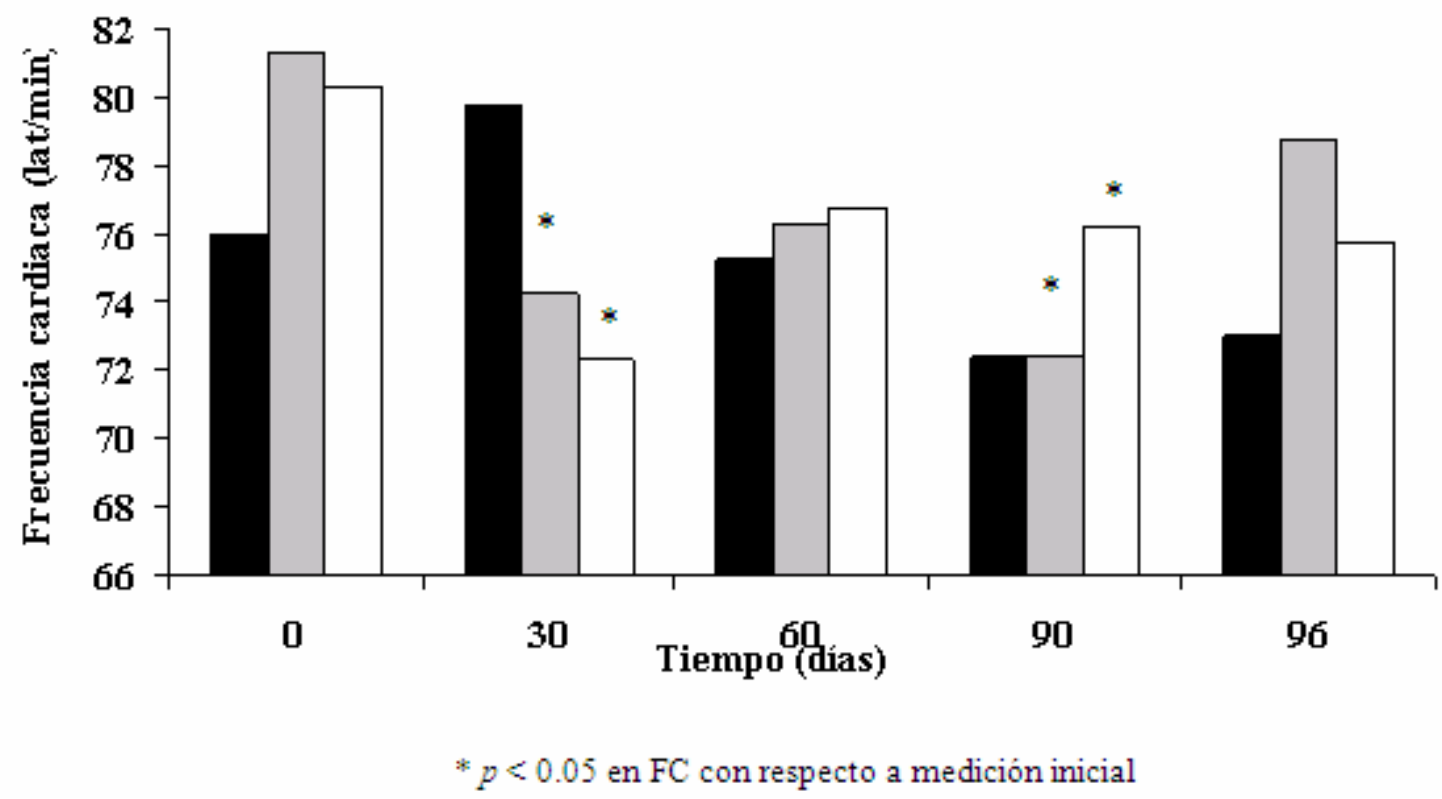

Figura 4. Valores de la frecuencia cardiaca en reposo a través del tiempo en adultos mayores.

Otro resultado importante que se obtiene de este estudio es que se redujo el estrés del sistema cardiovascular en reposo en este grupo de adultos mayores durante el programa. EL DP, un parámetro que mide el estrés del sistema cardiovascular, presentó reducciones a partir de 4 semanas de entrenamiento, independientemente de la intensidad del ejercicio (i.e., $30 \%$ o $70 \%$ de $5 \mathrm{RM}$ ), como se ilustra en la Figura 7, lo que indica que en reposo, el sistema cardiovascular trabaja con menor estrés, mientras se realiza el programa de ejercicio contra resistencia.

En comparación con el DP obtenido antes de comenzar el programa de ejercicios contra resistencia, estas reducciones son evidentes a las 4 semanas, mostrando una reducción del 18\% del estrés al sistema cardiovascular en reposo.

De manera similar, a las 8 semanas se presentó con una reducción del 12\%, y a las 12 semanas, hubo una reducción del 17\%. Estos hallazgos muestran una rápida adaptación del sistema cardiovascular en adultos mayores sanos, la cual se mantuvo durante el programa de ejercicios. Según Williams et al. (2007), un mecanismo que podría explicar la reducción en el stress hemodinámico podría ser el aumento en la fuerza muscular, la cual ciertamente ocurrió en los grupos que realizaron ejercicio. Sin embargo, la rápida adaptación en esta 
población podría explicarse debido al factor neuroendocrino, pues se ha comprobado que la norepinefrina se asocia positivamente a la edad; y así como lo demostró Duncan et al. (1985), las personas con mayores niveles de esta sustancia tienen una mayor reducción en los niveles de PAS, por tanto podría ser ésta una explicación al fenómeno de la rápida adaptación que experimentaron los participantes. Otra explicación podría ser atribuida al aumento en la capacidad de contracción del músculo cardíaco, debido al estímulo del ejercicio que se estaba realizando simultáneamente en ese período de tiempo.

- Control $\square \mathrm{GBO} \% \square \mathrm{G} 70 \%$

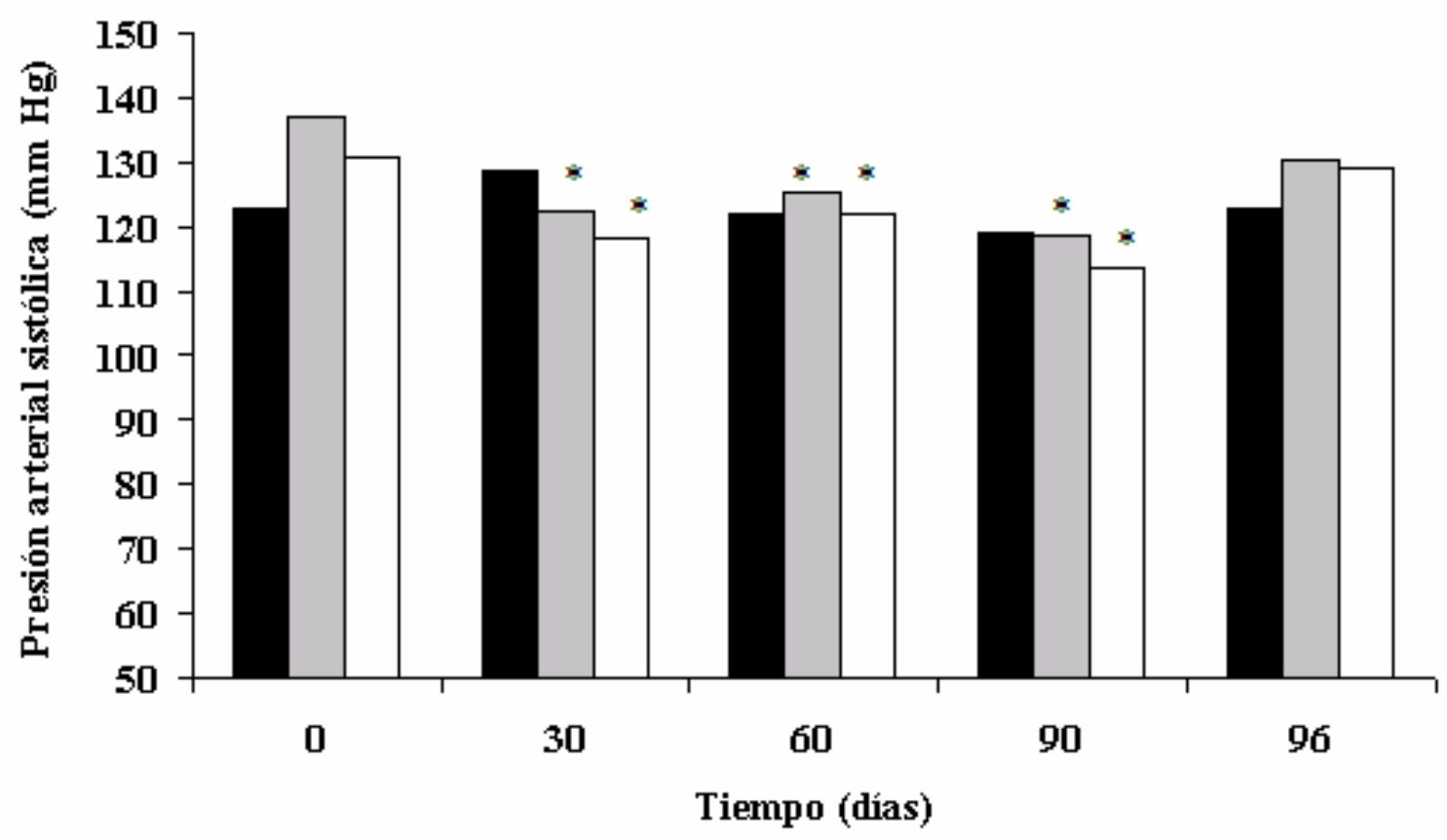

Figura 5. Valores de la presión arterial sistólica en reposo a través del tiempo en adultos mayores.

* $p<0.05$ en PAS con respecto a medición inicial

Otro hallazgo relevante de este estudio, es que el grado de estrés y el consumo de oxígeno del sistema cardiovascular regresa a valores similares a los iniciales incluso 6 días después de haber finalizado el programa de ejercicios, lo que indica la pérdida de las adaptaciones positivas del ejercicio físico en un periodo de tiempo muy corto (Figura 7).

Una fortaleza de este estudio es que se contó con un número aceptable de participantes. Otras investigaciones (Hagerman, Walsh, Staron, Hikida, Gilders, Murray, Toma, y Ragg, 2000; Ades, Ballor,
Ashikaga, Utton, y Sreekumaran-Nair, 1996) han basado sus hallazgos en muestras de 18 o 24 participantes. A pesar de que no se realizó un estudio a priori de la potencia para estimar el tamaño de la muestra, el número elevado de participantes en este estudio apoya la potencia de las pruebas estadísticas utilizadas. Esperamos que este trabajo pueda brindar información acerca de los cambios esperables en las variables hemodinámicas medidas que puedan servir para el cálculo del tamaño de la muestra de futuras investigaciones. 


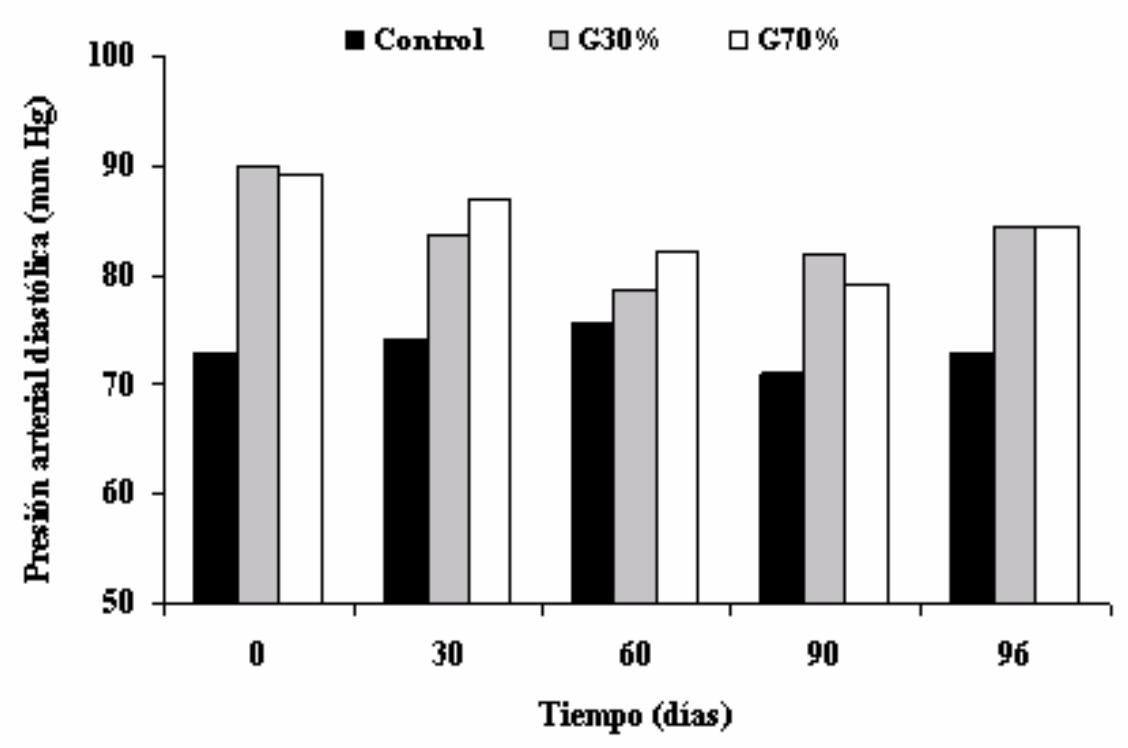

Figura 6. Valores de la presión arterial diastólica en reposo a través del tiempo en adultos mayores.

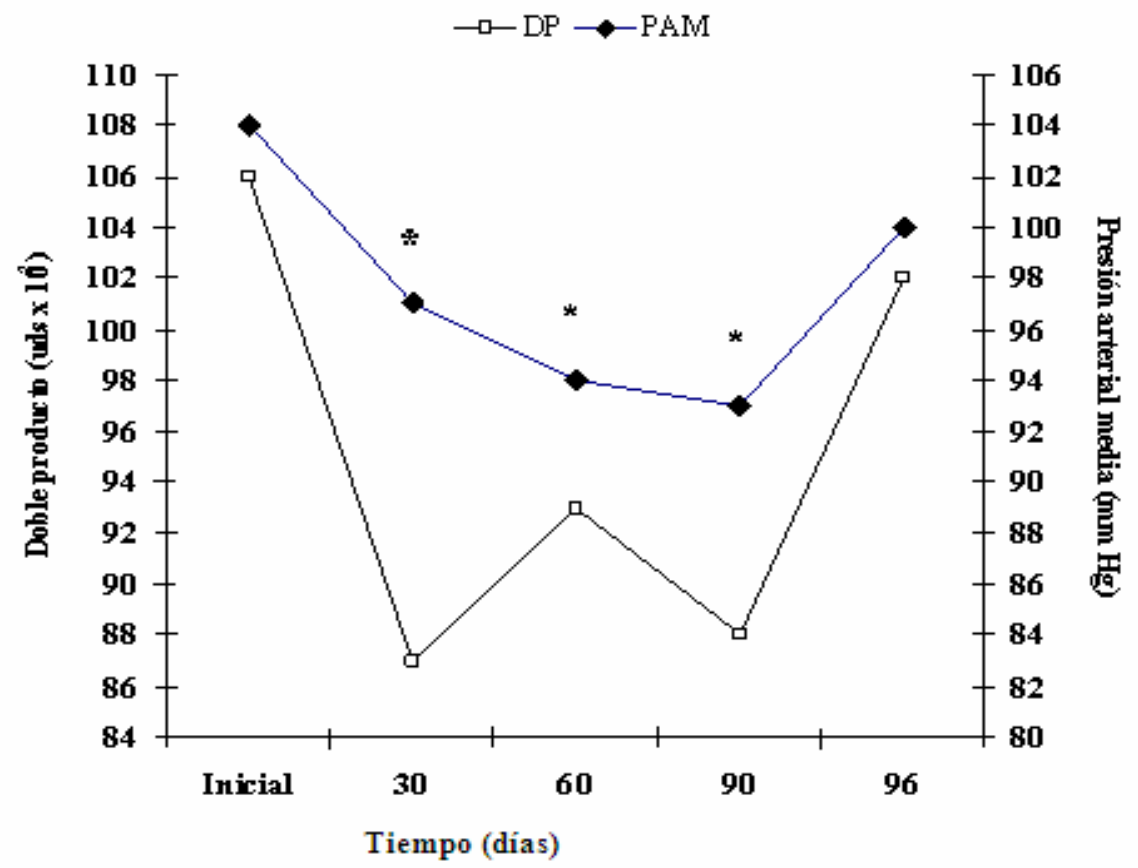

Figura 7. Adaptaciones hemodinámicas de la presión arterial media (PAM) y del doble producto (DP) en adultos mayores que participaron en un programa de entrenamiento contra resistencia de 12 semanas. ${ }^{*} p<0.05$ en PAM con respecto a medición inicial.

En conclusión, el ejercicio contra resistencia al $30 \%$ y $70 \%$ de 5RM, con una carga total de ejercicio entre 872.7 y 890.9 kg (1920 y $1960 \mathrm{lbs})$, respectivamente, no presenta niveles de PAS, PAD, FC y PAM de riesgo cardiovascular en adultos mayores de 60 años sin riesgo cardiovascular.
Además, el programa aplicado mejora el acondicionamiento cardiovascular en reposo durante el programa de ejercicios contra resistencia; sin embargo, estos beneficios se pierden a los 6 días de finalizado el programa de ejercicios, lo cual indica que una población con estas características debe 
tener un programa de actividad física constante. Se recomienda realizar investigaciones con las mismas intensidades de ejercicio en programas de mayor duración y determinar el proceso de pérdida de la adaptación de otras variables, incluyendo factores bioquímicos, biomecánicos y fisiológicos.

\section{Agradecimiento}

Se agradece al M.Sc. José Moncada Jiménez, del Laboratorio de Ciencias del Movimiento Humano LACIMOV -, por el apoyo brindado en la revisión del manuscrito.

\section{REFERENCIAS}

Ades, P. A., Ballor, D. L., Ashikaga, T., Utton, J. L., \& Sreekumaran-Nair, K. (1996). Weight training improves walking endurance in healthy elderly persons. Annals of Internal Medicine, 124(6), 568-572.

Bermon, S., Rama, D., y Dolisi, C. (2000). Cardiovascualr tolerance of health elderly subjects to weight-lifting exercises. Medicine \& Science in Sports \& Exercise, 32(11), 18451848.

Braith, R.W., y Stewart, K.J. (2006). Resistance training: Its role in the prevention of cardiovascular disease. Circulation, 113(22), 2642-2650.

Brown, A., Mc Cartney, N., y Sale, D. (1981). Positive adaptations to weight-lifting trainning in the elderly. Journal of Applied Physiology, $69,1725-1733$.

Duncan, J. J., Farr, J. E., Upton, S. J., Hagan, R. D., Oglesby, M. E., y Blair, S. N. (1985). The effects of aerobic exercise on plasma cathecolamines and blood pressure in patients with mild essential hypertention. Journal of the American Medical Association, 254(18), 26092613.

Fiatarone, M., Marcks, E., Ryan, N., Meredith, C., y Evans, W. (1990). High-intesity strength trainning in nonagenarians: Effect on skeletal muscle. Journal of the American Medical Association, 263, 3029-3034.
Fleck, S. (1988). Cardiovascular adaptations to resistance training. Medicine \& Science in Sports \& Exercise, 20, s146-s151.

Fleck, S. (1992). Cardiovascular response to strength training. En Koomi, P. (Ed), Muscular basics of strength and power in sport. Boston, MA: Blackwell.

Frontera, W., Meredith, C., O'Reilly, K., Knuttgen, H., y Evans, W. (1988). Strength conditioning in older men: Skeletal muscle hypertrophy and improved function. Journal of Applied Physiology, 64, 1038-1044.

Goldberg, A. (1989). Aerobic and resistance exercise modify risk factor for coronary heart disease. Medicine \& Science in Sports \& Exercise, 21, 669-674.

Green, J. S. \& Crouse, S.F. (1995). The effects of endurance training on functional capacity in the elderly: A meta-analysis. Medicine and Science in Sports and Exercise, 27(6), 920-926.

Hagberg, J. M., Montain, S. J., Martin, W. H. $3^{\text {rd }}$., y Ehsani, A. A. (1989). Effect of Exercise Trainning in 60 to 69 year-old persons with essential hypertension. American Journal of Cardiology, 64(5), 348-353.

Hagerman, F. C., Walsh, S. J., Staron, R. S., Hikida, R. S., Gilders, R. M., Murray, T. F., Toma, K., \& Ragg, K. E. (2000). Effects of high-intensity resistance training on untrained older men. I. Strength, cardiovascular, and metabolic responses. Journal of Gerontology, 55A(7), B336-B346.

Heyn, P., Abreu, B. C., \& Ottenbacher, K. J. (2004). The effects of exercise training on elderly persons with cognitive impairment and dementia: A meta-analysis. Archives of Physical Medicine and Rehabilitation, 85(10), 1694-1704.

Hill-Westmoreland, E. E., Soeken, K., \& Spellbring, A. M. (2002). A meta-analysis of fall prevention programs for the elderly: how effective are they? Nursing Research, 51(1), 18. 
Israel, S. (1992). Age-related changes in strength and special groups. En Koomi, P. (Ed), Muscular basics of strength and power in sport. Boston, MA: Blackwell.

Joint National Committee on Prevention, Detection, Evaluation, and Treatment of High Blood Pressure (2003). Seventh Report of the Joint National Committee on Prevention, Detection, Evaluation, and Treatment of High Blood Pressure JNC Express (NIH Publication No. 03-5233). Bethesda, MD: U.S. Department of Health and Human Services.

Kaver, R., Fonseca, L., y Salazar, W. (1999). Efecto del entrenamiento de un programa de ejercicios con pesas, en la máquina de extensión de rodillas y en el press de banca, con cargas de $30 \%$ ó $70 \%$ de $5 \mathrm{RM}$ en el adulto mayor. Revista de Gerontología, 1(1), 49-61.

Lopopolo, R. B., Greco, M., Sullivan, D., Craik, R. L., \& Mangione, K. K. (2006). Effect of therapeutic exercise on gait speed in community-dwelling elderly people: A metaanalysis. Physical Therapy, 86(4), 520-540.

MacDougall, J. D., Tuxen, D., Sale, D. G., Moroz, J. R., y Sutton, J. R. (1985). Arterial blood response to heavy resistance exercise. Journal of Applied Physiology, 58, 785-790.

Madden, K. M., Levy, W. C., y Stratton, J. K. (2006) Exercise training and heart rate variability in older adult female subjects. Clinical and Investigative Medicine, 29(1), 2028.

Miller, R. (1994). The biology of aging and longevity. Principles of geriatric medicine and gerontology. New York, NY: Mc Graw-Hill.

Pescatello, L. S., Fargo, A. E., Leach, C. N. Jr, y Scherzer, H. H. (1990). Short-term effect of dynamic exercise on arterial blood pressure. Circulation, 83(5), 1557-1561.

Province, M. A., Hadley, E. C., Hornbrook, M. C., Lipsitz, L. A., Philip, M. J., Mulrow, C. D., Ory, M. G., Sattin, R. W., Tinetti, M. E., \&
Wolf, S. L. (1995). The effects of exercise on falls in elderly patients: A preplanned metaanalysis of the FICSIT trials. Journal of the American Medical Association, 273(17), 13411347.

Sale, D. G., Moroz, D. E., McKelvie, R. S., MacDougall, J. D., y McCartney, N. (1993). Comparison of blood pressure response to isokinetic and weight-lifting exercise. European Journal of Applied Physiology and Occupational Physiology, 67(2), 115-120.

Stone, M.H., Fleck, S.J., Triplett, N.R. \& Kraemer, W.J. (1991). Physiological Adaptations resistance training exercise. Sports Medicine, $11,210-231$.

Williams M. A., Haskel W.L., Ades, P.A., Amsterdam, E.A., Bittner, B., Franklin, B.A., Gulanick, M., Laing, S.T., y Steward, K.J. (2007). Resistance exercise in individual with and without cardiovascular disease: 2007 Update: A scientific statement from the American Heart Association Council on Clinical Cardiology and Council on Nutrition, Physical Activity and Metabolism. Circulation, 116, 572-584. 